\title{
IgG4-assoziierte Aortitis bei einem sechsjährigen Jungen
}

IgG4-RD (IgG4-related diseases) sind in den Formenkreis inflammatorisch fibrosierende Systemerkrankungen einzuordnen und können unterschiedlichste Organsysteme befallen. Charakteristisch ist eine lymphoplasmatische Infiltration von IgG4-positven Plasmazellen in das betroffene Organsystem sowie ein erhöhtes Serum-IgG4 [1]. Die genaue Pathogenese ist bislang ungeklärt. Es wird unter anderem diskutiert, dass über molekulares Mimikry die Th2und Treg-Zellen stimuliert werden, was u. a. die Ausschüttung von Th2- und Treg-assoziierten Zytokinen wie IL-6 begünstigt. Diese induzieren im weiteren Verlauf IgG4-B-Zellen, was zu einer lgG4-Antikörperinfiltration und Organfibrose führt. Die lymphoplasmatische Infiltration von IgG4 prägt das charakteristische pathologische Bild der Erkrankung [2, 3].

Die Inzidenz im Kindesalter ist aufgrund der bislang fehlenden systematischen Erfassung nicht zu eruieren [4]. Im japanischen Raum liegt die geschätzte Inzidenz bei Erwachsenen zwischen 0,28-1,08/100 000 . Dieses entspricht ungefähr 336-1300 Neuerkrankungen pro Jahr [5].

Im Kindes- und Jugendalter ist die häufigste Manifestationsform der Pseudotumor Orbitae, gefolgt von Pankreatitiden oder Cholangitiden. Beschrieben sind unter anderem auch Manifestationen in der Lunge, Schilddrüse, Speichel-/Tränendrüse sowie in Form einer fibrosierenden Mediastinitis. Darüber hinaus wurde wiederholt eine begleitende interstitielle Nephritis beobachtet [1]. Die allgemeinen Diagnosekriterien lassen eine Diagnosestellung auf Basis der klinisch/serologischen Befunde, klinisch/histologischen Befunde aber auch auf Grundlage der serologisch/histologischen Befunde zu oder auch rein auf Grundlage der Histologie (siehe Kasten „Diagnosekriterien") [4].

Zur Remissionsinduktion hat sich Prednison bewährt. Dieses kann in reduzierter Dosis auch als Erhaltungstherapie gegeben werden. Insbesondere im Kindesalter ist eine steroidsparende Immunsuppression anzustreben mit entweder Azathioprin, Methotrexat, Mycophenolat-Mofetil oder Rituximab, die je nach Aktivität der Erkrankung,
Grundvoraussetzungen des Patienten und Erfahrung des betreuenden Arztes gewählt werden kann [4].

\section{Anamnese}

Ein sechsjähriger kinderkardiologischer Patient osteuropäischer Herkunft wird bei subjektivem Wohlbefinden zur Mitbeurteilung in der Kinderrheumatologie vorgestellt. Zuvor wurde im Rahmen einer Aortenteilresektion mit Prothesenersatz bei Mehrstufenstenose anhand des intraoperativ gewonnenen histologischen Materials der Verdacht auf eine Großgefäßvaskulitis gestellt.

Anamnestisch waren Schwangerschaft und Geburt unauffällig gewesen, die entwicklungsneurologische Reifung war regelrecht verlaufen. Der Junge hatte in den ersten zwei Lebensjahren rezidivierende Analabszesse. Im Alter von drei Jahren wurde eine solitäre Nierenzyste mit Harntransportstörung marsupialisiert und ebenfalls ein frühkindliches Asthma bronchiale diagnostiziert. Mit fünf Jahren fiel in der Vorsorgeuntersuchung eine arterielle Hypertonie auf, als dessen Ursache sich eine hochgradige Mehrstufenstenose der thorakalen und abdominellen Aorta herausstellte. Bei zum Teil kritischen Stenosen wurde die Indikation zur (partiellen) operativen Korrektur mit Aorteninterponat gestellt.

\section{Diagnostik}

Differenzialdiagnostisch wurden bei Großgefäßvaskulitis folgende Erkrankungen in Betracht gezogen: Takayasu-Arteriitis, Polyarteriitis nodosa inkl. Adenosin-Deaminase (ADA)-2-Defizienz, IgG4-related disease, Morbus Behçet, das Cogan-Syndrom sowie eine Treponemeninfektion.

Die einzige laborchemische Auffälligkeit war ein erhöhter IgG4-Spiegel mit erhöhten IgG4/lgG- und IgG4/lgG1-Ratios. Die Inflammationsparameter (C-reaktives Protein, Blutsenkung und Leukozyten) waren bei Vorstellung in der rheumatologischen Ambulanz normwertig. Antinukleäre Antikörper oder Antikörper gegen neutrophile Granulozyten (ANCA) waren nicht nachweisbar und eine Komplementaktivierung

\section{DIAGNOSEKRITERIEN}

Allgemeine Diagnosekriterien für IgG4-RD

1. Diffuse oder fokale Vergrößerung/ Raumforderung in einem/ mehreren Organen

2. Erhöhung des Serum-IgG4> $135 \mathrm{mg} / \mathrm{dl}$ oder $>1,5 \mathrm{x}$ der oberen Norm

3. Histologische Veränderungen:

a. Organ-Infiltration mit Lymphozyten/Plasmazellen mit Fibrose

b. Infiltration IgG4-positiver Plasmazellen ins Gewebe (>10/GF)

c. und/oder lgG4-/lgG-Verhältnis $>40 \%$

d. geschichtete/gewirbelte Fibrose

e. obliterative Phlebitis

Die Diagnose kann bei Erfüllen folgender Kriterien gestellt werden:

$1+2$ oder $1+3 a / b$ oder $2+3 a / b$ oder $3 a / b / c / d$.

Histologische Kriterien für IgG4-RD Major-Kriterien

- dichte lymphoplasmazelluläre Infiltrate

- Fibrose, in der Regel geschichtet

Minor-Kriterien

- obliterative Phlebitis

- Gewebs-Eosinophilie

Die Diagnose kann bei Erfüllen folgender Kriterien gestellt werden:

- 2 Major-Kriterien

- 1 Major-Kriterium + 2 Minor-Kriterien + lgG4-/lgG-Verhältnis > 50\% oder > 10 lgG4-Zellen/Gesichtsfeld.

Klinische Kriterien für IgG4-RD

- Schwellung oder Dysfunktion in einem/mehreren Organen (charakteristisch für lgG4-RD)

- Typische radiologische Veränderungen für IgG4-RD

- IgG4-Serumtiter > 1,5 x über dem oberen Normwert

nach [4] 

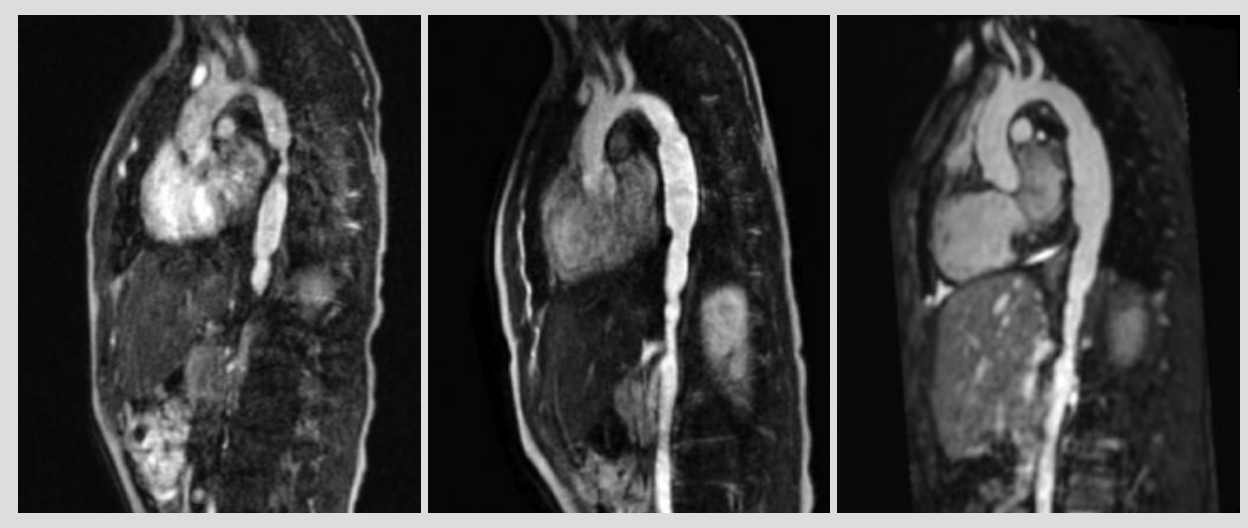

- Abb. 1 Bild links: MRT Thorax-Aorta präoperativ bei Verdacht auf Midaortic-Syndrom; Bild Mitte: MRT Thorax-Aorta postoperativ (Aorteninterponat) vor Beginn der immunsuppressiven Therapie; Bild rechts: MRT Thorax-Aorta drei Monate nach Beginn der immunsuppressiven Therapie.

bestand zu keinem Zeitpunkt. Eine histologische Nachuntersuchung des Aortenresektats zeigte in der multiplen Myeloma Oncogene 1 (MUM1)-Immunhistochemie nur vereinzelte MUM1-positive Plasmazellen, wobei ein geringer Teil dieser sich auch positiv in der IgG4-Immunhistochemie färbte.

\section{Therapie}

Anhand der allgemeinen und klinischen Diagnosekriterien kann bei Vorliegen einer inflammatorischen abdominellen Aortitis und entsprechender IgG4-Erhöhung die Diagnose einer lgG4-assoziierten Erkrankung gestellt werden, allerdings in diesem Fall bei Nichterfüllen der histologischen Kriterien.

Nach Diagnosestellung wurde eine Therapie mit Prednison $0,8 \mathrm{mg} / \mathrm{kg} K G=20 \mathrm{mg} / \mathrm{d}$ absolut für zwei Wochen begonnen, gefolgt von einer Reduktion auf $0,6 \mathrm{mg} / \mathrm{kg}$ $\mathrm{KG}=15 \mathrm{mg} / \mathrm{d}$ absolut für weitere zwei Wochen. Anschließend wurde monatlich um $2,5 \mathrm{mg} / \mathrm{d}$ bis zu einer Erhaltungsdosis von $2,5 \mathrm{mg}$ absolut reduziert. Zeitgleich mit dem Beginn der Steroidtherapie wurde mit Methotrexat (MTX) 13,2 mg/m² Körperoberfläche/Woche subkutan begonnen.

\section{Verlauf}

Nach Beginn der immunsuppressiven Therapie hatte der Junge im MRT der Aorta einen stabilen Befund. Serologisch zeigte sich ein deutlicher Abfall des lgG4s und

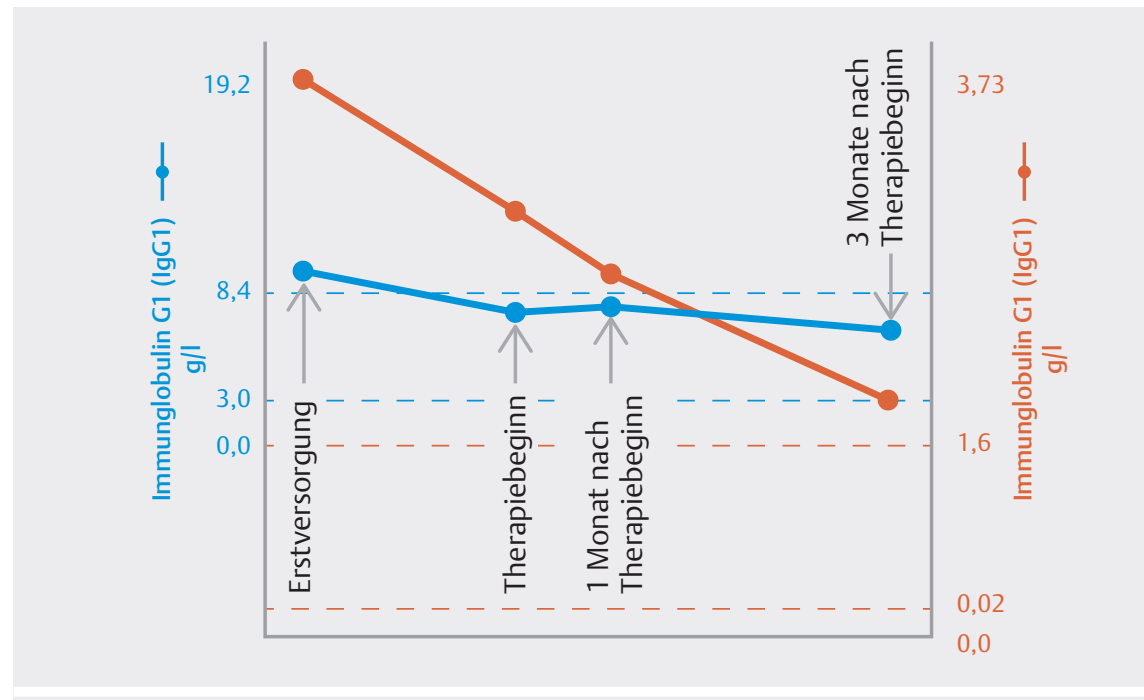

- Abb. 2 Verlauf von IgG1 und IgG4 vor und nach Beginn der immunsuppressiven Therapie mit systemischen Steroiden und Methotrexat (initial IgG4: IgG1-Ratio=0,39; im Verlauf nach Beginn der Therapie $\lg \mathrm{G} 4$ : $\lg \mathrm{G} 1-$ Ratio $=0,23$ ).

konsekutiv eine Normalisierung der lgG4/ IgG-Ratio und lgG4/lgG1-Ratio.

Begleitend zum Beginn der immunsuppressiven Therapie entwickelte der Patient als neues Symptom eine Hyperthyreose ohne nachweisbare Antikörper oder sonografische Auffälligkeiten der Schilddrüse. Hier wurde daraufhin eine Carbimazol-Therapie initiiert. Drei Monate nach Beginn der Carbimazol-Therapie war die Hinzunahme von L-Thyroxin notwendig, um eine euthyreote Stoffwechsellage einzustellen. Zu keinem Zeitpunkt war ein reduzierter Allgemeinzustand oder eine sonstige Einschränkung im Alltag beobachtet worden.

\section{Diskussion}

Wir berichten hier über einen sechsjährigen Jungen mit der Erstmanifestation einer Aortitis als lgG4-RD. Bislang ist dies der erste publizierte Fall im Kindesalter. Die Diagnose wurde anhand der klinischen und allgemeinen Diagnosekriterien gestellt mit einer erhöhten lgG4/lgG-Ratio und entsprechender Organveränderung mit histologisch gesicherter Aortitis.

Ob die anderen geschilderten Symptome mit rezidivierenden Analabszessen, Nierenzyste, das vorbeschriebene frühkindliche Asthma bronchiale sowie die Hyperthyreose Teil dieses Krankheitsbildes sind, bleibt unklar. Zumindest wäre die Hyperthyreose 


\section{ZUSAMMENFASSUNG \\ Hintergrund \\ IgG4-related disease (IgG4-RD) ist eine inflammatorisch fibrosieren- de Systemerkrankung, die sich in unterschiedlichsten Organsystemen manifestieren kann und einer im- munsuppressiven Therapie bedarf. Im Kindes- und Jugendalter handelt es sich um eine Rarität.}

\section{Fall}

Bei einem Sechsjährigen wurde nach einer Aortenteilresektion mit Prothesenersatz anhand des hier gewonnenen histologischen Materials der Verdacht auf eine Großgefäßvaskulitis gestellt. Anamnestisch bestanden in den ersten zwei Lebensjahren rezidivierende Analabszesse. Im Alter von drei Jahren wurde eine solitäre Nierenzyste mit konsekutiver Harntransportstörung diagnostiziert und marsupialisiert. Mit fünf Jahren fiel ein arterieller Hypertonus auf, bei dessen Abklärung eine hochgradige Mehrstufenstenose der thorakalen und der abdominellen Aorta diagnostiziert wurde. Laborchemisch zeigten sich deutlich erhöhte lgG4-Spiegel. Im sechsten Lebensjahr entwickelte der Junge begleitend zum Beginn einer immunsuppressiven Therapie eine Hyperthyreose.

\section{Schlussfolgerung}

Anhand der allgemeinen und klinischen Diagnosekriterien kann in diesem Fall bei Vorliegen einer inflammatorischen abdominellen Aortitis und entsprechender lgG4-Erhöhung die im Kindesalter äußerst seltene Diagnose einer IgG4-assoziierten Erkrankung gestellt werden, allerdings bei Nichterfüllen der histologischen Kriterien.

als frühes Zeichen einer sich entwickelnden entzündlich fibrosierenden Veränderung der Schilddrüse denkbar. Zumal die Riedel-Thyreoiditis eine häufige Manifestationsform von IgG4-RD im Kindesalter ist [1].

Das Besondere an dem vorliegenden Fall ist die Erstbeschreibung einer Aortitis im Rahmen von IgG4-RD bei einem Kind. Diese
Manifestationsform ist normalerweise eine nur im Erwachsenalter beobachtete Verlaufsform [6].

Bislang sind keine geeigneten Aktivitätsparameter noch gute Möglichkeiten zur Unterscheidung zwischen möglicher Krankheitsaktivität und bleibenden Organschäden für die lgG4-RD bei Kindern beschrieben. In einer retrospektiven Evaluation von 40 erwachsenen Patienten mit Periaortitis haben Mizushima et al. beispielsweise einen Rückgang der periaortalen Dicke im Schnitt 30 Monate nach Beginn einer immunsuppressiven Therapie gesehen [7].

Beim IgG4-Spiegel wird vermutet, dass es bei Patienten, die bei Diagnosestellung ein erhöhtes Serum-IgG4 hatten, dieses durchaus die Krankheitsaktivität widerspiegeln kann [8]. Somit kann bei unserem Patienten der stetige Abfall des Serum-lgG4s und ein drei Monate nach Beginn der Therapie stabiler Befund im MRT ( $\triangleright$ Abb. 1, \ Abb. 2) als Behandlungserfolg gewertet werden. Jedoch sind weitere kinderrheumatologische Verlaufskontrollen notwendig und der weitere Verlauf sollte abgewartet werden.

\section{FAZIT}

In der Pädiatrie gibt es seltene bzw. bisher nicht beschriebene Manifestationsformen von IgG4-assoziierten Autoimmunerkrankungen. Aufgrund der Seltenheit ist es sehr schwer, eine solche Diagnose zu stellen bzw. verschiedene Symptome der Erkrankungsgruppe zuzuordnen. Nach aktuellem Wissenstand bleibt stets eine Restunsicherheit über die Richtigkeit der Diagnose und der dementsprechenden Therapieoptionen.

\section{Interessenkonflikt}

Alle Autoren bestätigen, dass kein Interessenkonflikt im Zusammenhang mit diesem Artikel besteht.

\section{Einhaltung ethischer Richtlinien}

Der Patient und seine Eltern haben ihr schriftliches Einverständnis für die Publikation dieses Manuskripts gegeben.

\section{Autorinnen/Autoren}

Youssef $\mathrm{H}$; Lutz T; Helling-Bakki A

Zentrum für Kinder- und Jugendmedizin, Angelika-Lautenschläger-Klinik, Heidelberg

\section{Korrespondenzadresse}

\section{Dr. Hudda Youssef}

Zentrum für Kinder- und Jugendmedizin, Angelika-Lautenschläger-Klinik, Kinderheilkunde I, Kinder- und Jugendrheumatologie, Im Neuenheimer Feld 430, 69120 Heidelberg

Hudda.Youssef@med.uni-heidelberg.de

\section{Literatur}

[1] Karim F et al. IgG4-related disease: a systematic review of this unrecognized disease in pediatrics. Pediatr Rheumatol Online J 2016; 14 (1): 18

[2] Okazaki $\mathrm{K}$ et al. Recent advances in the concept and diagnosis of autoimmune pancreatitis and IgG4-related disease. J Gastroenterol 2011; 46 (3): 277-288

[3] Kasashima $S$ et al. Upregulated interleukins (IL-6, IL-10, and IL-13) in immunoglobulin G4-related aortic aneurysm patients. J Vasc Surg 2018; 67 (4): 1248-1262

[4] Strothmann JML, Hennecke P, Hufnagel M. IgG4-related disease (IgG4-RD) - auch eine Erkrankung des Kindesalters. arthritis + rheuma 2013; 33 (5): 317-322

[5] Umehara $\mathrm{H}$ et al. IgG4-related disease and its pathogenesis-cross-talk between innate and acquired immunity. Int Immunol 2014; 26 (11): 585-595

[6] Ozawa $\mathrm{M}$ et al. Clinical features of IgG4-related periaortitis/periarteritis based on the analysis of 179 patients with IgG4-related disease: a case-control study. Arthritis Res Ther 2017; 19 (1): 223

[7] Mizushima I et al. Clinical course after corticosteroid therapy in IgG4-related aortitis/ periaortitis and periarteritis: a retrospective multicenter study. Arthritis Res Ther 2014; 16 (4): R156

[8] Wallace ZS et al. An International, Multi-Specialty Validation Study of the IgG4-Related Disease Responder Index. Arthritis Care Res (Hoboken) 2018

\section{Bibliografie}

DOI https://doi.org/10.1055/a-0824-5417 arthritis + rheuma 2019; 39: 54-56 (c) Georg Thieme Verlag KG Stuttgart · New York ISSN 0176-5167 